\title{
Robust Watermarking of 3D Polygonal Meshes
}

\author{
Han Sae SONG ${ }^{\dagger a)}$, Student Member and Nam Ik $\mathrm{CHO}^{\dagger \mathrm{b})}$, Nonmember
}

\begin{abstract}
SUMMARY This paper presents an algorithm for the robust watermarking of 3D polygonal mesh models. The proposed algorithm embeds the watermark into a 2D image extracted from the 3D model, rather than directly embedding it into 3D geometry. The proposed embedding domain, i.e., the $2 \mathrm{D}$ image, is devised to be robust against the attacks like mesh simplification which severely modifies the vertices and connectivity while preserving the appearance of the model. The watermark-embedded model is obtained by using a simple vertex perturbation algorithm without iterative optimization. Two exemplary watermark applications using the proposed methods are also presented: one is to embed several bits into 3D models and the other is to detect only the existence of a watermark. The experimental results show that the proposed algorithm is robust against similarity transform, mesh simplification, additive Gaussian noise, quantization of vertex coordinates and mesh smoothing, and that its computational complexity is lower than that of the conventional methods.

key words: information security, digital watermarking, robust watermarking, 3D polygonal mesh model
\end{abstract}

\section{Introduction}

A watermark is a sort of hidden information that can be used in many applications such as ownership protection, authentication, fingerprinting and labeling [1]-[3]. The watermark for ownership protection is secretly hidden so that it could be extracted only by the owner who has the right key. The watermark for authentication works as the unique signature of digital data. If the digital data are tampered with, the inserted watermark is also spoiled, and the tampering can be detected. The watermark for fingerprinting works as the unique identifier of each data recipient, which is used for tracing illegal distributions. The watermark for labeling is used to deliver useful information such as the serial number, and product name.

This wide range of watermarking applications requires different and sometimes conflicting properties [2]-[4]. The distinct properties of watermarking algorithms are usually described by several criteria: robustness, perceptibility and the blindness of detection [2], [3], [5]. The robustness of watermarking means the difficulty of removing the watermark. The watermarking algorithms for ownership protection are required to be robust, whereas those for authentication are required to be fragile. Perceptibility describes whether the embedded watermark can be noticed by human sensors such as eyes and ears. The imperceptible watermarking is prefer-

Manuscript received September 6, 2007.

${ }^{\dagger}$ The authors are with School of Electrical Engineering and Computer Science, Seoul National University, Korea.

a) E-mail: merlin @ispl.snu.ac.kr

b)E-mail: nicho@snu.ac.kr

DOI: 10.1093/ietisy/e91-d.5.1512 able for hiding the presence of a watermark, whereas the perceptible one is used for discouraging unauthorized duplications or for degrading commercial value. Blindness indicates the need for original data to retrieve a watermark. Watermarking algorithms are non-blind if the watermark detection procedures need original data, and they are blind if do not need such data.

This paper focuses on watermarking for threedimensional polygonal mesh models. 3D mesh is one of the most popular methods for representing 3D objects, which is described by vertices and edges. We propose a new approach to the robust watermarking of 3D polygonal mesh models, which is basically blind, but the robustness increases if the original model is given. Both the blind and non-blind algorithms are discussed in this paper. The proposed algorithm embeds the watermark into the virtual range data of the 3D model and only the vertex coordinates are modified by the embedding process. It is devised to be robust particularly against simplification attacks, but it is shown to be robust against other attacks as well. To better clarify the scope of this paper as well as its main concerns, the existing methods and the possible attacks to 3D mesh data are summarized in the following.

\subsection{Previous Researches}

There are several watermarking algorithms for 3D mesh models. Ohbuchi et al. proposed three watermarking algorithms for polygonal mesh models [6]. One method inserts a message by adjusting ratios of edge lengths and the ratio of triangle height over its base. Another method embeds a message by adjusting ratios of tetrahedral volumes. Yet another method makes a visible pattern by subdividing selected faces and the pattern becomes visible with wireframe rendering. Ohbuchi et al. also proposed a robust watermarking using mesh Laplacian matrix [7], [8]. Each vertex is projected to each eigenvector of the Laplacian matrix and the coefficient vectors are produced. The watermark is inserted by modifying these coefficient vectors. The eigenvectors correspond to frequency bands and the proper frequency bands can be selected according to the kinds of attack.

Benedens proposed a robust watermarking algorithm using normal vectors [9]. Normal vectors that have similar directions are grouped and projected onto 2D local coordinates. Then, the centroid of the projected vertices is moved according to a message bit. Finally, vertex coordinates are 
perturbed to obtain the watermark-embedded normal vectors. Benedens also introduced high-capacity watermarking schemes for labeling [10].

Praun et al. proposed a robust watermarking by extending the spread spectrum method to 3D data [11]. They identify great geometric changes and construct surface bases by using progressive mesh algorithm. Then, vertices are perturbed by using global displacement directions and scalar weighting functions for the surface bases. When the model is simplified, resampling which recovers the original connectivity is used to detect the watermark.

Kanai et al. developed a frequency-domain 3D watermarking [12]. They decomposed a 3D mesh model into the wavelet coefficient vectors and the coarsest mesh. The norms of the wavelet vectors are modified by watermarking and the watermarked model is obtained by using inverse wavelet transformation. Yeo et al. introduced an authentication algorithm for 3D models [13], using two hash functions. Indexes derived from vertex coordinates and the centroids of neighboring vertices are used as inputs for the hash functions.

\subsection{Attacks to 3D Mesh Models}

An attack is any kind of operation that can remove a watermark. It is either a common 3D processing methods or a malicious one. The common processing methods include basic 3D operations such as rotation, translation, scaling, deformation and cropping, as well as 3D processing algorithms such as smoothing, mesh simplification and compression. The malicious attacks include noise addition, connectivity modification and vertex reordering. Among these operations, mesh simplification is one of the most challenging attacks, in that it reduces the number of vertices while preserving the appearance of 3D model. Moreover, it severely changes vertex coordinates and connectivity. Hence, it is challenging to detect the watermark, when models are attacked through the mesh simplification.

Several algorithms have been proposed in previous researches to handle the mesh simplification [8], [9], [11], [14], [15]. These algorithms can be roughly categorized into two approaches. The first uses embedding domains that have a certain degree of invariance against mesh simplification. The embedding domain consists of features extracted from the 3D model. The watermark is embedded into the extracted features, rather than directly embedded into the $3 \mathrm{D}$ geometry. Then, the watermarked model is obtained by using the inverse process of feature extraction, i.e., conversion from the watermark-embedded features to the 3D structure (vertices and edges). An example of the robust embedding domain is the statistics (Extended Gaussian Image) of normal vectors of 3D mesh used in [9]. It is based on the observation that the directions of normal vectors are not much affected by the change of vertices and connectivity. The watermark is embedded into normal vectors, and the vertices are perturbed so that the $3 \mathrm{D}$ model would have watermarked normals.
The second approach recovers the original connectivity by using "resampling" or "remeshing" [8], [11], [14], [15]. Resampling is a process to fit the original mesh to the suspected one, while minimally deforming it [11]. After resampling, the original mesh becomes the resampled one which has similar geometry to the watermarked model and the same connectivity as the original. Hence, the watermark is detected from this resampled model. Resampling, however, is highly complex because it involves ray-polygon intersection problem as well as cost minimization. Moreover, blind detection is not possible with this approach when the mesh is attacked by simplification.

\section{Proposed Method for 3D Mesh Watermarking}

The proposed method is thus based on the first approach: the embedding domain approach for non-blind as well as blind watermarking. It is shown herein that the proposed embedding domain is more robust to the simplification attack than the existing ones. The embedding domain presented in this paper has an explicit relationship with vertices, and thus the change of the domain caused by the embedded watermark is easily reflected to vertex coordinates without any optimization process.

A 2D image extracted from the 3D mesh is used as the embedding domain. Each pixel value of the $2 \mathrm{D}$ image is a distance measured from the surface of the given 3D model to each reference point that is cylindrically positioned around the 3D model. This image is similar to the output of a 3D scanner that uses a LASER ranging device. For this reason, it is referred to as range image in this paper. The use of the range image as the embedding domain, brings with it the following advantages of robustness and computational efficiency: (1) robustness to attacks that change both vertices and connectivity, such as mesh simplification, (2) easy inversion process: an explicit method exists to perturb the vertices without optimization processes, (3) no need for resampling: watermark is detected without resampling even if vertices and connectivity are changed, and (4) easy adoption of 2D image watermarking: the embedding domain is a $2 \mathrm{D}$ image and many existing $2 \mathrm{D}$ algorithms can be easily adopted.

The entire flow of the proposed algorithm is briefly described as follows. To embed the watermark, the range image is first obtained from the original 3D model. Then the watermark is embedded into the range image by using a 2D image watermarking algorithm. The watermarked 3D model is finally obtained by vertex perturbation which reflects the changes of the range image to vertex coordinates. In the detection process, the range image is obtained from the suspicious model, and it is also obtained from the original in the case of non-blind detection. Then, the watermark is retrieved by the detection procedure of the image watermarking used in the embedding process. The details of the detection will be described later. 


\section{Range Image Extraction}

Range image extraction consists of three steps: defining a cylinder around the mesh model, positioning reference points on the cylinder and then calculating distances from these reference points to the surface of the mesh. These distances become the pixel values of the range image.

The parameters of bounding cylinder are central axis, center point, radius and height. Two important factors in the preparation of cylinder parameters are: the best pose of cylinder for the reference points and the invariance under rotation, scaling and translation (RST). The best pose means that when the vertices of the mesh are radially projected onto the central axis of cylinder, the projected vertices should have the maximum variance. Such a central axis can be found by the principle component analysis (PCA) of vertices, which enables us to see the largest part of a 3D model from the side face of the cylinder.

For the invariance under the rotation of 3D model, a local coordinate system is defined by using three eigenvectors. For the translation invariance, the mean vector of all vertices is set to be the origin of the local coordinate. Also, the radius and height of the cylinder are set to be tightly fit to the 3D model, which gives invariance under scaling.

Figure 1 shows the bounding cylinder and its parameters. $\mathbf{m}_{\mathbf{v}}$ is the mean vector of all the vertices, which is used as the origin of local coordinate system. The eigenvectors $\mathbf{s}_{\mathbf{1}}, \mathbf{s}_{\mathbf{2}}, \mathbf{s}_{\mathbf{3}}$ are used as the local axes. Radius and height are denoted as $r$ and $h$. The detailed explanation and equations for the parameters are described in Appendix A.

Reference points are regularly positioned on the side face of the cylinder. For integer indexing of the reference points, a $u-v$ coordinate is defined on the side face of the cylinder as shown in Fig. 1. The $u$-axis has the same direction as $\mathbf{S}_{1}$, and the $v$-axis follows the circumference of cylinder. The reference points are indexed by using the index set, $\left\{\left(u_{r}, v_{r}\right) \mid u_{r}=0, \cdots, N_{u}-1, v_{r}=0, \cdots, N_{v}-1\right\} . N_{v}$ and $N_{u}$ are the numbers of reference points in the direction of $v$ and $u$, which determine the size of a range image that is related to the capacity of watermark. The detail of size decision is described in Appendix B.

The global coordinate of each reference point $\left(u_{r}, v_{r}\right)$ can be calculated by following equations. The local coordinate of an arbitrary $(u, v)$ is
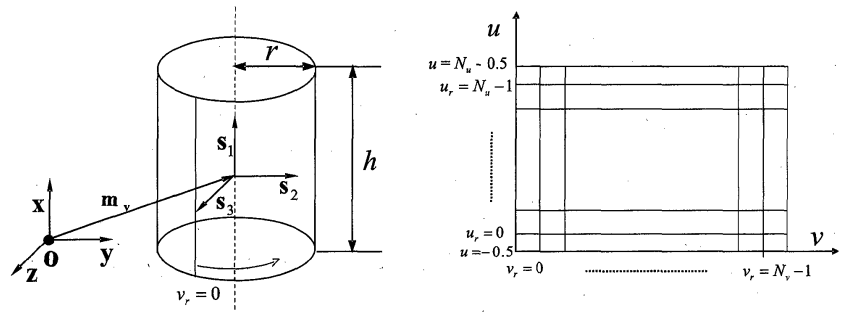

Fig. 1 Configuration of the bounding cylinder and unrolled view of $u-v$ plane defined on the side face of the cylinder.

$$
\begin{aligned}
& \left(s_{1}, s_{2}, s_{3}\right) \\
& \quad=\left((u+0.5) \frac{h}{N_{u}}+h_{\min }, r \sin \frac{2 \pi v}{N_{v}}, r \cos \frac{2 \pi v}{N_{v}}\right)
\end{aligned}
$$

with respect to the eigenvector axes $\mathbf{s}_{\mathbf{1}}, \mathbf{s}_{\mathbf{2}}, \mathbf{s}_{\mathbf{3}}$, and its global coordinate is

$$
(x, y, z)=\mathbf{m}_{\mathbf{v}}+s_{1} \mathbf{S}_{\mathbf{1}}+s_{2} \mathbf{S}_{\mathbf{2}}+s_{3} \mathbf{S}_{\mathbf{3}} .
$$

The pixel values of range image are distances from each reference point to the surface of the 3D model. The distance can be obtained by drawing a straight line from each reference point $\left(u_{r}, v_{r}\right)$ to the surface of model along the radial direction of cylinder, and then by measuring the distance from the reference point to the intersecting point on the face of 3D model. Calculation of distance from a point to an intersecting polygon has already been studied intensively in literatures such as [16]-[19]. We adopt the uniform space subdivision method [18], [19] for the fast computation.

If 3D models have cylindrical or spherical topology, the range image can cover almost the entire area of 3D surfaces. If not, it may not cover the whole surface due to occlusions. If the cylindrical occlusion occurs, multiple intersection points exist for one reference point. A solution to this occlusion problem is to consider all the multiple intersections as the independent target of watermarking. That is, the range image can have several pixel values like color image. For simplicity, the shortest distance, i.e., the distance from the reference point to the outermost face is used as a pixel value of the range image in the experiments. If there is no intersection between the straight line and the surface, we mark that point as "invalid pixel". The invalid pixels are ignored in the watermarking process.

As a simple example to demonstrate the robustness of the range image, two range images are compared; one is obtained from the original model and the other from the simplified mesh that has one eighth number of original vertices. The histogram of pixel differences of two images is shown in Fig. 2. The horizontal axis means the values of pixel difference and the vertical axis is the number of pixels within each bin. It is observed that the differences are concentrated near zero, which means that the range image is not much

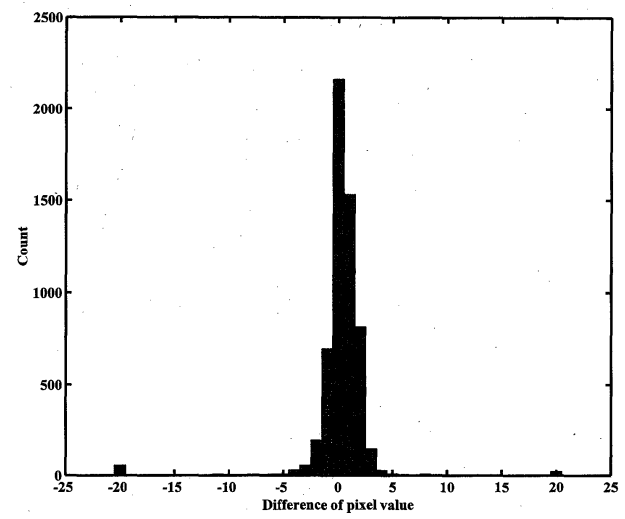

Fig. 2 Histogram of pixel differences between the range image of the original model and that of the model attacked by $1 / 8$ simplification. 
affected by the $1 / 8$ mesh simplification attack.

\section{Watermark Embedding}

The watermark is embedded into the range image, and then the vertices are adjusted according to the watermarked image. The vertex adjustment results in the watermarked 3D model.

\subsection{Range Image Watermarking}

Any existing 2D watermarking algorithm can be used for embedding the watermark into the range image. Two typical cases of watermarking algorithms are considered in this paper. One embeds several message bits and the other inserts a random vector and detects only the existence of watermark. DCT-based blind algorithm is adopted from [20] and the non-blind algorithm is derived by slightly modifying this blind algorithm.

The pixels of a range image are distances in 3D space and thus its dynamic range is different for various 3D models. For consistent embedding, the range image is normalized to the range $[0,255]$ before embedding, and recovered to the original scale after embedding.

DCT-based watermarking: First, the rectangular region of valid pixels is selected and divided into several $8 \times 8$ blocks. Then, each block is transformed by the discrete cosine transform (DCT). For each DCT block, the watermark is embedded into thirteen AC coefficients by adding weighted watermark signal to the coefficients as $z_{i}=c_{i} \pm \alpha w_{i}$, where the sign of addition depends on the message bits. The value of $\alpha$ adjusts the strength of watermark which controls the robustness and visibility, and $w_{i}$ is a random signal of \pm 1 . The seed value to generate this random signal and the selected region for DCT are used as the key. The watermarked range image is obtained by performing IDCT of watermarked coefficients and inverse normalization.

In the detection phase for non-blind scheme, a message bit is derived for each DCT block by using $w_{i}{ }^{*}=\left(z_{i}-c_{i}\right) / \alpha$. Then, the correlation between the original and extracted watermark is calculated as

$$
\rho=\frac{\sum_{i}\left(w_{i}^{*}-\overline{w_{i}^{*}}\right)\left(w_{i}-\overline{w_{i}}\right)}{\sqrt{\sum_{i}\left(w_{i}^{*}-\overline{w_{i}^{*}}\right)^{2}} \sqrt{\sum_{i}\left(w_{i}-\overline{w_{i}}\right)^{2}}}
$$

where the upper bar means average value. If $\rho>0$, the message bit in this block is decoded as 1 . Otherwise, the bit is decoded as 0 .

In the case of blind detection, likelihood ratio test is employed from [20]. For simplicity, pure watermark that carries no message is considered and the embedding equation becomes $z_{i}=c_{i}+\alpha w_{i}$, where the sign of addition is always positive. As described in [20], the simplified loglikelihood for this case is

$$
l(z)=\sum_{i} \beta^{c}\left(\left|z_{i}\right|^{c}-\left|z_{i}-\alpha w_{i}\right|^{c}\right)
$$

where $c=1$ and $\beta=\sqrt{6} / \sigma_{i}$, assuming that the distribution of each DCT coefficient is Laplacian with standard deviation $\sigma_{i}$.

Spatial domain watermarking: A watermark vector $\mathbf{w}$ is embedded in the pixel domain of the range image. The elements of watermark vector $\mathbf{w}$ are Gaussian random numbers with zero mean and unit variance. The seed number that generates these random numbers is used as the key.

The watermark is embedded into the normalized range image $I(x, y)$ by adding a scaled and translated version of a sinc function. That is, the watermarked image is

$$
\begin{aligned}
& \forall(x, y) \in\left\{(0,0), \cdots,\left(N_{v}-1, N_{u}-1\right)\right\}, \\
& \tilde{I}(x, y)=I(x, y) \\
& \quad+\alpha \sum_{i=1}^{N_{w}} w_{i} f\left(x-x_{i}, y-y_{i}\right)\left|\frac{I\left(x_{i}, y_{i}\right)-127.5}{127.5}\right|
\end{aligned}
$$

where $\alpha$ is the watermark strength, $w_{i}$ is the $i$-th component of the watermark vector, $f(\cdot)$ is the sync function, $N_{w}$ is the number of elements of $\mathbf{w}$ and $\left(x_{i}, y_{i}\right)$ for $i=1, \cdots, N_{w}$ are center points of the function $f(x, y)$. The $2 \mathrm{D}$ sinc function is defined as

$$
f(x, y)= \begin{cases}\frac{\sin \left(2 \pi \sqrt{x^{2}+y^{2}} / R_{0}\right)}{2 \pi \sqrt{x^{2}+y^{2}} / R_{0}} & \text { if } \sqrt{x^{2}+y^{2}}<R_{0} \\ 0 & \text { otherwise, }\end{cases}
$$

where $R_{0}$ determines the size of main lobe, and is set to $R_{0}=\sqrt{\frac{N_{u} N_{v}}{\pi N_{w}}}$ in this application, in order to prevent the function $f\left(x-x_{i}, y-y_{i}\right)$ from overlapping with the ones in other positions. The positions of $\left(x_{i}, y_{i}\right)$ are determined to be distributed in the region of valid pixels of the range image with proper intervals. The exact position of $\left(x_{i}, y_{i}\right)$ is not so significant as long as they are not too close to each other. If the pixel is invalid, no watermark is embedded in it.

The watermarked image in Eq. (5) can be written in the matrix form as

$$
\tilde{\mathbf{p}}=\mathbf{p}+\mathbf{A w}
$$

where $\mathbf{p}$ and $\tilde{\mathbf{p}}$ are $N_{v} N_{u} \times 1$ column vectors of pixels, $\mathbf{A}$ is a $N_{v} N_{u} \times N_{w}$ matrix and $\mathbf{w}$ is a $N_{w} \times 1$ column vector. Specifically, $\left(x+y \times N_{v}\right)$-th components of $\mathbf{p}$ and $\tilde{\mathbf{p}}$ are equal to $I(x, y)$ and $\tilde{I}(x, y)$ respectively, and the $\left(x+y \times N_{v}, i\right)$-th element of $\mathbf{A}$ is $\alpha f\left(x-x_{i}, y-y_{i}\right)\left|\frac{I\left(x_{i}, y_{i}\right)-127.5}{127.5}\right|$. Since $\mathbf{A}$ is not a rectangular matrix, the least-squares method is used to extract the watermark $\mathbf{w}$ for the given $\mathbf{p}$. The extracted watermark $\mathbf{w}^{*}$ is

$$
\mathbf{w}^{*}=\left(\mathbf{A}^{\mathbf{T}} \mathbf{A}\right)^{-1} \mathbf{A}^{\mathbf{T}}(\tilde{\mathbf{p}}-\mathbf{p})
$$

\subsection{Vertex Perturbation}

The final step of watermark embedding is to make the watermarked 3D model that corresponds to the watermarked $2 \mathrm{D}$ range image. That is, the vertices are moved to match the distance changes induced by the watermark embedding. 
If the distance from the reference point to the surface of the model is decreased by the watermark, the vertices around the intersecting point should be moved toward the corresponding reference point, and vise versa. Vertex perturbation needs two steps. First, all the vertices are partitioned into several sets of vertices, where each set includes the neighboring vertices of each intersecting point. Second, the modified distance is applied to only the vertices in the set that are associated with the corresponding distance.

For determining the sets of vertices, each vertex is projected to the $u-v$ grid of side face. The $u-v$ coordinate of each vertex $\mathbf{v}$ is calculated using Eqs. (1) and (2), which is denoted by $u(\mathbf{v})$ and $v(\mathbf{v})$. Note that the $u$ coordinate is unique, but two $v$ coordinates are possible because a vertex can be projected to the side face in two radial directions. The direction which results in the positive inner product with the normal of vertex $\mathbf{v}$ is selected.

Then, each vertex is inserted into the set that is associated with the nearest reference point in the $u-v$ plane. With these projected coordinates of vertices, we define a set of vertices that is associated with the reference point $\left(u_{r}, v_{r}\right)$ as

$$
\begin{array}{r}
\mathcal{B}\left(u_{r}, v_{r}\right)=\left\{\mathbf{v}: \mathbf{v} \in \mathcal{V} \text { and }\left|u(\mathbf{v})-u_{r}\right|<0.5\right. \\
\text { and } \left.d\left(v(\mathbf{v}), v_{r}\right)<0.5\right\}
\end{array}
$$

where $\mathcal{V}$ is the set of all the vertices and $d(x, y)$ is a metric that defines the distance between two coordinates in the $v$ axis. Since $v$ axis is circular, we define this metric as

$$
d(x, y)=\min \left(|x-y|,|| x-y\left|-N_{v}\right|\right) .
$$

The vertices in $\mathcal{B}\left(u_{r}, v_{r}\right)$ are now perturbed by using the change of the distance that is defined as

$$
\Delta l\left(u_{r}, v_{r}\right)=\tilde{l}\left(u_{r}, v_{r}\right)-l\left(u_{r}, v_{r}\right)
$$

where $\tilde{l}\left(u_{r}, v_{r}\right)$ is the pixel value of the watermarked range image and $l\left(u_{r}, v_{r}\right)$ is that of the original. The straightforward method of perturbation is to move vertices along the radial direction by the amount of change. That is, the modified vertex $\mathbf{v}^{\prime}$ for each vertex $\mathbf{v} \in \mathcal{B}\left(u_{r}, v_{r}\right)$ is described as

$$
\mathbf{v}^{\prime}=\mathbf{v}-\Delta l\left(u_{r}, v_{r}\right) \mathbf{r}(v(\mathbf{v}))
$$

where $\mathbf{r}(\cdot)$ is a radial direction vector pointing given $v$ coordinate which is defined as

$$
\mathbf{r}(x)=\cos \frac{2 \pi x}{N_{v}} \mathbf{s}_{\mathbf{3}}+\sin \frac{2 \pi x}{N_{v}} \mathbf{s}_{\mathbf{2}} .
$$

But this modification makes discontinuity at the boundary between the vertex sets. Thus, a weighting factor is used to smooth the discontinuity. The weighting factor gives less perturbation to the vertices if they are closer to the boundary of the set. The neighboring $\Delta l$ is also considered for smoothness. To be precise, the weighting factor is defined as

$$
\begin{aligned}
& w\left(u_{r}, v_{r}, u, v\right) \\
& \quad=\left\{\begin{array}{c}
\frac{\sin \left(\pi\left|u-u_{r}\right|\right) \sin \left(\pi d\left(v, v_{r}\right)\right)}{\left(\pi^{2}\left|u-u_{r}\right| d\left(v, v_{r}\right)\right)} \\
\text { if }\left|u-u_{r}\right|<1 \text { and } d\left(v, v_{r}\right)<1 \\
0, \quad \text { otherwise, }
\end{array}\right.
\end{aligned}
$$

and the weighted change of distance is calculated as

$$
\begin{aligned}
& \Phi\left(u_{r}, v_{r}, u, v\right) \\
& \quad=\sum_{j=-1}^{1} \sum_{k=-1}^{1} w\left(u_{r}+j, v_{r}+k, u, v\right) \Delta l\left(u_{r}+j, v_{r}+k\right) .
\end{aligned}
$$

Finally, instead of Eq. (12), the vertex modification is performed as

$$
\begin{aligned}
& \forall\left(u_{r}, v_{r}\right) \text { and } \quad \forall \mathbf{v} \in \mathcal{B}\left(u_{r}, v_{r}\right), \\
& \mathbf{v}^{\prime}=\mathbf{v}-\Phi\left(u_{r}, v_{r}, u(\mathbf{v}), v(\mathbf{v})\right) \mathbf{r}(v(\mathbf{v})) .
\end{aligned}
$$

\section{Watermark Detection}

The proposed algorithm supports both blind and non-blind detection depending on the availability of the original 3D model. Also, the 2D watermarking for the range image can be blind or non-blind depending on whether the range image of the original mesh is needed or not. Hence, four possible combinations of watermark detection modes exist:

3D non-blind / 2D non-blind: The original 3D model and the original range image are needed. The original range image is obtained from the original model and the watermarked range image is calculated from the watermarked model. Then, the watermark is detected by the detection procedure of the $2 \mathrm{D}$ watermarking algorithm. The watermarked range image is obtained by using the original axes and cylinder. Since the vertices of watermarked model are perturbed, the eigenvectors calculated from the watermarked model are similar to, but not the same as the original. Hence, the original axes result in more accurate calculation of range image. If the watermarked model is transformed with RST, the watermarked model is aligned with the original by using mesh registration [21] in order to use the original axes and cylinder.

3D non-blind / 2D blind: This case is the same as above except that $2 \mathrm{D}$ watermarking of range image is blind. But this choice seems unreasonable because the original 2D range image is available from the given original mesh model and thus it is unnecessary to use blind 2D watermarking method. 3D blind / 2D non-blind: The original model is not given, but the $2 \mathrm{D}$ range image watermarking needs the original range image. Hence, the original range image should be transmitted in order to detect watermark. It will increase the size of the detection key but it is much smaller in size than the entire 3D model. In this case, the original eigenvectors are not available and the mesh registration is also not possible. The eigenvectors computed from the watermarked model are used as axes for reference points, which results in less robust detection.

3D blind / 2D blind: The original model is not given and the original range image is also not needed. The original eigen- 
vectors are not available and the axis error is unavoidable. We detect watermark without any original information, and this case is the most fragile among the four combinations.

\section{Experimental Results}

Figure 3 shows 3D models used in the experiments and cxamples of attacks. Happy Buddha is downloaded from Stanford 3D Scanning Repository [22] and Blade is from Georgia Tech's Large Geometric Models Archive [23]. David is from Digital Michelangelo Project [24].

\subsection{Robustness Test}

The attacks considered in the experiments are mesh simplification, additive Gaussian noise, quantization of vertex coordinate and surface smoothing. The mesh simplification reduces the number of vertices by $1 / 2$ and $1 / 8$ of the original, where 'plycrunch' algorithm is used. The Gaussian noise attack adds a random vector to each vertex. Each component of the random vector is a sample of an independent Gaussian random variable with zero mean and unit variance. The noise vectors are adjusted to have the same magnitude, which is denoted by the ratio of the magnitude to the longest diagonal length of bounding box. In the experiments, the magnitude ratios of $0.03 \%$ and $0.1 \%$ are considered. The quantization attack is to quantize each element of coordinates by 11 bit resolution. More precisely, the bounding box is divided uniformly by $2048 \times 2048 \times 2048$ grid and every vertex is replaced by the nearest intersection point of the grid.

\section{D non-blind / 2D non-blind case DCT-based watermarking:}

The parameters used for the experiments are listed in Table 1 . Note that the watermark is embedded into the rectangular region of 'Size', with the left corner at the 'Corner' in $(u, v)$ coordinate. The keys of the watermark for the proposed algorithm are $N_{u}, N_{\nu}$, 'Corner', 'Size', $\alpha$ and the secd of the random signal generator. The proposed algorithm is compared with Benedens' [9] for every model. For Benedens' algorithm, the number of inserted bits are set to be the same as that of the proposed algorithm. The number of bins is determined twice as large as that of inserted bits, and half of the bins are used for embedding. For fair comparison, the overall distortion caused by watermark is controlled to be almost the same in every cases. METRO algorithm [25] is used to calculate the distortion between the original models and watermarked models. For the proposed algorithm, the watermarked models are first rotated, translated and scaled, and then the attacks are applied. Table 2 shows the average bit error rates (ratio of the number of error bits to the number of inserted bits) of 200 different watermarks. It is observed that the average bit error rate of the proposed algorithm is less than half of that of the Benedens' algorithm. Spatial domain watermarking:

The size of range image $\left(N_{u}, N_{v}\right)$ for the spatial domain watermarking is the same as in Table 1 . The length of the watermark vector is set to be $50\left(N_{w}=50\right)$. The keys of the watermark are $N_{u}, N_{v}, \alpha$, the embedding matrix in Eq. (7) and the seed of random number generator. The proposed scheme is compared with Praun et al.'s algorithm [11], where the

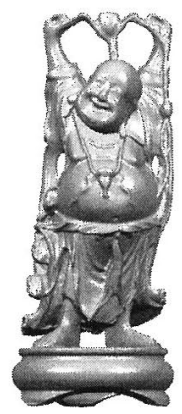

(a)Happy Buddha

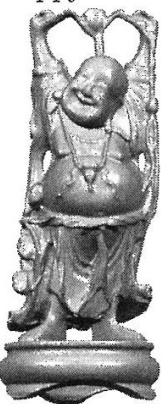

(f) Simplified $1 / 2$ \# of vertices

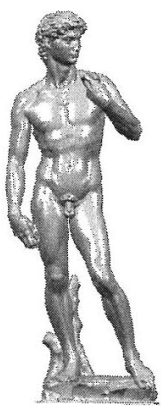

(b) David

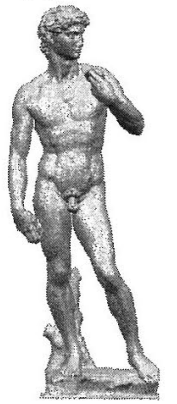

(g) Noise $0.03 \%$

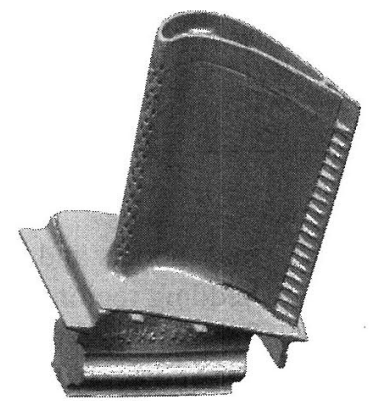

(c) Blade

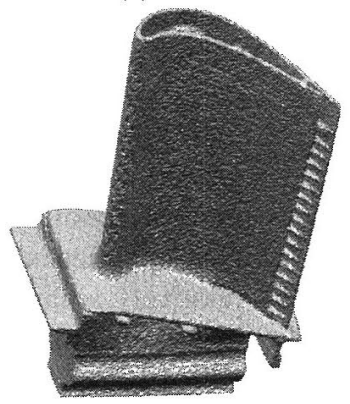

(h) Noise $0.1 \%$

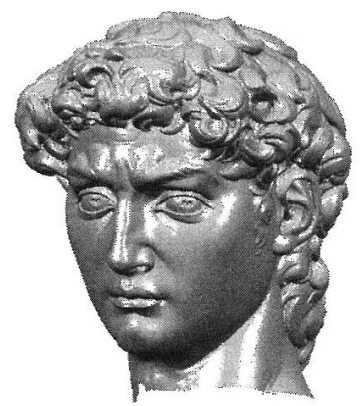

(d) Head of David

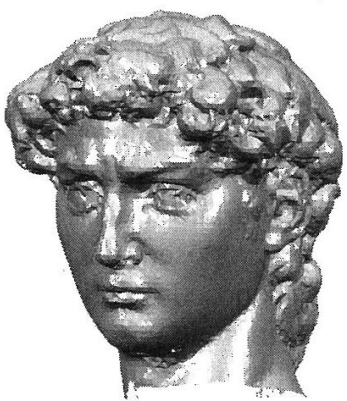

(i) Simplified $1 / 8$ \# of vertices

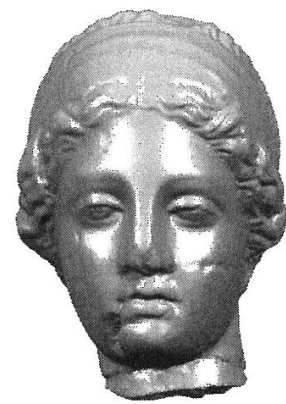

(e) Venus

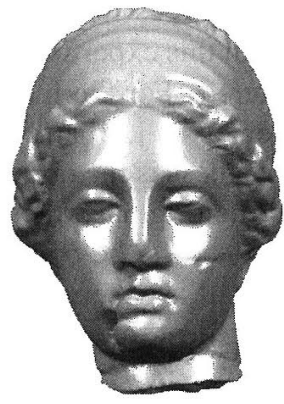

(j) Smoothed

Fig. 3 3D models for the cxperiments and various attacks. 
Table 1 Parameters for the DCT-based algorithm.

\begin{tabular}{ccccccc}
\hline Model & \# of Vertex & $N_{u} \times N_{v}$ & $\begin{array}{c}\text { Corner } \\
(u, v)\end{array}$ & $\begin{array}{c}\text { Size } \\
u \times v\end{array}$ & $\begin{array}{c}\text { Capacity } \\
\text { (bits) }\end{array}$ & $\alpha$ \\
\hline HB & 148488 & $80 \times 66$ & $(20,1)$ & $56 \times 56$ & 49 & 0.3 \\
BL & 144650 & $64 \times 57$ & $(8,0)$ & $56 \times 56$ & 49 & 0.1 \\
DA & 162774 & $127 \times 69$ & $(1,0)$ & $64 \times 64$ & 64 & 0.3 \\
HE & 151855 & $69 \times 102$ & $(8,0)$ & $40 \times 96$ & 60 & 0.3 \\
VE & 50002 & $35 \times 68$ & $(1,0)$ & $32 \times 64$ & 32 & 0.2 \\
\hline
\end{tabular}

* $\mathrm{HB}=$ Happy Buddha, $\mathrm{BL}=$ Blade, $\mathrm{DA}=$ David, $\mathrm{HE}=$ David $\mathrm{Head}, \mathrm{VE}=$ Venus

Table 2 Bit error rates of the proposed algorithm using the DCT-based 2D watermarking and Benedens' algorithm.

\begin{tabular}{ccccccc}
\hline Model & Watermark & SIM 1/2 & SIM 1/8 & N 0.03\% & N 0.1\% & Q 11 bit \\
\hline HB & DCT & $0.26 \%$ & $18.6 \%$ & $2.16 \%$ & $5.40 \%$ & $0.19 \%$ \\
& Benedens & $2.06 \%$ & $41.6 \%$ & $1.04 \%$ & $45.7 \%$ & $1.22 \%$ \\
BL & DCT & $6.28 \%$ & $33.0 \%$ & $1.18 \%$ & $6.38 \%$ & $3.38 \%$ \\
& Benedens & $22.9 \%$ & $48.2 \%$ & $4.27 \%$ & $44.5 \%$ & $5.56 \%$ \\
DA & DCT & $7.80 \%$ & $11.1 \%$ & $1.09 \%$ & $4.13 \%$ & $1.90 \%$ \\
& Benedens & $4.45 \%$ & $35.9 \%$ & $9.11 \%$ & $47.7 \%$ & $13.2 \%$ \\
HE & DCT & $1.97 \%$ & $11.3 \%$ & $0.29 \%$ & $1.30 \%$ & $0.26 \%$ \\
& Benedens & $5.70 \%$ & $38.8 \%$ & $0 \%$ & $35.3 \%$ & $1.42 \%$ \\
VE & DCT & $0.03 \%$ & $5.20 \%$ & $0.16 \%$ & $0.31 \%$ & $0.66 \%$ \\
& Benedens & $2.94 \%$ & $37.5 \%$ & $0 \%$ & $11.7 \%$ & $0 \%$ \\
Avg. & DCT & $3.27 \%$ & $15.8 \%$ & $0.98 \%$ & $3.50 \%$ & $1.28 \%$ \\
& Benedens & $7.61 \%$ & $40.2 \%$ & $2.89 \%$ & $37.0 \%$ & $4.28 \%$ \\
\hline
\end{tabular}

* SIM=simplification, $\mathrm{N}=$ Gaussian noise attack, $\mathrm{Q}=$ quantization attack

Table $3 \rho$ of the proposed algorithm using spatial 2D watermarking and Praun's.

\begin{tabular}{cccccccc}
\hline Model & Watermark & N 0.03\% & N 0.1\% & Q 11 bit & SIM 1/2 & SIM 1/8 & Taubin Smooth \\
\hline HB & spatial & 0.73 & 0.49 & 0.83 & 0.87 & 0.55 & 0.84 \\
& Praun & 0.95 & 0.78 & 0.99 & 0.59 & 0.18 & 0.90 \\
BL & spatial & 0.88 & 0.71 & 0.94 & 0.91 & 0.47 & 0.88 \\
& Praun & 1.00 & 0.95 & 1.00 & 0.69 & 0.14 & 0.69 \\
DA & spatial & 0.62 & 0.36 & 0.88 & 0.74 & 0.62 & 0.73 \\
& Praun & 0.97 & 0.83 & 1.00 & 0.44 & 0.49 & 0.97 \\
HE & spatial & 0.81 & 0.44 & 0.88 & 0.69 & 0.35 & 0.66 \\
& Praun & 0.95 & 0.76 & 0.98 & 0.76 & 0.24 & 0.82 \\
VE & spatial & 0.99 & 0.92 & 0.99 & 0.99 & 0.93 & 0.99 \\
& Praun & 0.97 & 0.95 & 0.97 & 0.51 & 0.05 & 0.97 \\
Avg. & spatial & 0.81 & 0.58 & 0.91 & 0.84 & 0.58 & 0.82 \\
& Praun & 0.97 & 0.85 & 0.99 & 0.60 & 0.22 & 0.87 \\
\hline
\end{tabular}

watermark strength $\epsilon$ is set to be 0.01 . The watermark strength of proposed algorithm is adjusted so that the watermarked models of both algorithms have similar distortions when calculated by the METRO algorithm. The experiment is performed with RST attack. We apply rotations of 5,8 and 10 degrees to each axis and scale the models 3 times larger. In this experiment, the mesh smoothing attack is also considered. For the mesh smoothing, we use the same parameters as in [26] and perform 10 iterations. The measure of robustness is the normalized correlation ( $\rho$ in Eq. (3)) between the original watermark vector and the extracted one. Table 3 shows the correlation of proposed and Praun's algorithm. It is observed that the proposed algorithm is more robust against the mesh simplification attack, while Praun's algorithm is more robust against the other attacks.

\section{D blind / 2D blind case}

In this case, the watermark is detected without the orig- inal models and the original range images. The size of embedding region is the same as the non-blind DCT algorithm. Table 4 show the detection rate that is the ratio of the number of detected watermarks to the total number of embedded models. The detection threshold is zero, that is, the watermark is detected if $l(z)$ in Eq. (4) is greater than zero. It shows more than $80 \%$ successful detection for the noise attacks and filtering. For $1 / 2$ simplification, it shows more than $80 \%$, successful detection but the performance is degraded with $1 / 8$ vertices. Head and Venus model show particularly poor performance for simplification because they have spherical shape. The models with spherical shape have relatively ambiguous principle axes which are more sensitive to the errors of vertices.

\subsection{D Blind vs. 3D Non-blind Comparison}

3D blind and 3D non-blind performances are also compared, 
Table 4 Detection rate of blind case.

\begin{tabular}{ccccccc}
\hline Model & N 0.03\% & N 0.1\% & Q 11 bit & SIM 1/2 & SIM 1/8 & Taubin Smooth \\
\hline HB & $86 \%$ & $88 \%$ & $86 \%$ & $84 \%$ & $78 \%$ & $80 \%$ \\
BL & $100 \%$ & $86 \%$ & $100 \%$ & $100 \%$ & $26 \%$ & $96 \%$ \\
DA & $96 \%$ & $88 \%$ & $94 \%$ & $88 \%$ & $58 \%$ & $82 \%$ \\
HE & $94 \%$ & $98 \%$ & $94 \%$ & $10 \%$ & $8 \%$ & $94 \%$ \\
VE & $88 \%$ & $86 \%$ & $92 \%$ & $14 \%$ & $4 \%$ & $78 \%$ \\
\hline
\end{tabular}

Table 5 Blind vs. Non-blind: bit error rates of DCT-based algorithm.

\begin{tabular}{llllllll}
\hline Model & Watermark & N 0.03\% & N 0.1\% & Q 11 bit & SIM 1/2 & SIM 1/8 & Taubin Smooth \\
\hline HB & Non-blind & $7.80 \%$ & $25.84 \%$ & $3.06 \%$ & $9.27 \%$ & $31.51 \%$ & $9.51 \%$ \\
& Blind & $11.18 \%$ & $24.04 \%$ & $3.47 \%$ & $33.14 \%$ & $53.31 \%$ & $10.04 \%$ \\
BL & Non-blind & $12.57 \%$ & $38.98 \%$ & $2.73 \%$ & $15.35 \%$ & $58.37 \%$ & $11.55 \%$ \\
& Blind & $10.82 \%$ & $45.22 \%$ & $4.86 \%$ & $25.59 \%$ & $69.67 \%$ & $16.86 \%$ \\
DA & Non-blind & $11.78 \%$ & $27.16 \%$ & $5.53 \%$ & $12.69 \%$ & $23.44 \%$ & $14.66 \%$ \\
& Blind & $9.22 \%$ & $26.06 \%$ & $7.94 \%$ & $39.94 \%$ & $43.59 \%$ & $15.59 \%$ \\
HE & Non-blind & $2.67 \%$ & $9.97 \%$ & $2.60 \%$ & $5.87 \%$ & $22.40 \%$ & $6.53 \%$ \\
& Blind & $4.70 \%$ & $9.87 \%$ & $3.10 \%$ & $88.50 \%$ & $91.53 \%$ & $6.10 \%$ \\
VE & Non-blind & $0.00 \%$ & $1.75 \%$ & $1.69 \%$ & $0.00 \%$ & $7.88 \%$ & $0.00 \%$ \\
& Blind & $0.25 \%$ & $4.88 \%$ & $1.88 \%$ & $90.31 \%$ & $90.69 \%$ & $0.31 \%$ \\
Avg. & Non-blind & $6.96 \%$ & $20.74 \%$ & $3.12 \%$ & $8.63 \%$ & $28.72 \%$ & $8.45 \%$ \\
& Blind & $7.23 \%$ & $22.01 \%$ & $4.25 \%$ & $55.50 \%$ & $69.76 \%$ & $9.78 \%$ \\
\hline
\end{tabular}

Table 6 Processing time (in seconds, Intel Pentium4 1.7 GHz, 512Mbyte RAM).

\begin{tabular}{ccccccc}
\hline Model & Process & DCT & Spatial & Benedens & Praun(+Resampling*) & Registration \\
\hline HB & embed & 12.3 & 15.8 & 779.3 & 681.6 & - \\
& detect & 16.3 & 19.7 & 17.9 & $50.0(+249.7)$ & 11.9 \\
BL & embed & 11.8 & 13.9 & 433.4 & 828.7 & - \\
& detect & 18.4 & 16.3 & 17.9 & $48.3(+216.7)$ & 92.2 \\
DA & embed & 14.2 & 18.8 & 1470.7 & 503.8 & - \\
& detect & 15.0 & 23.2 & 24.3 & $52.8(+2839.1)$ & 105.6 \\
HE & embed & 13.5 & 17.2 & 2007.3 & 795.9 & - \\
& detect & 17.7 & 21.6 & 21.9 & $213.1(+304.8)$ & 348.0 \\
VE & embed & 4.2 & 5.2 & 158.6 & 1530.0 & - \\
& detect & 5.6 & 6.2 & 4.3 & $31.3(+64.2)$ & 10.2 \\
\hline
\end{tabular}

* Resampling is only needed for simplification attacks.

both with non-blind 2D. In the case of 3D blind, the original 2D range image needs to be transmitted as side information. The original axes are available only in the 3D nonblind case, and thus the experiment shows the effect of axis error with the fixed performance of 2D watermarking.

The errors in the recovery of axes result in the degraded performance of detection or the loss of 3D watermark. Hence, the accurate recovery of the reference axes is an important factor for the successful watermark detection. If the original model is given at the detection phase, the axes and cylinder parameters can be recovered from the original model instead of the watermarked one. If needed, mesh registration can be performed to align the original with the watermarked model. In the case of blind detection, the axes recovered from the watermarked or attacked mesh have inevitable errors.

The comparison of blind and non-blind schemes for DCT-based algorithm is given in Table 5. As expected, blind scheme shows higher bit errors than the non-blind one. The increase of bit error rate is large particularly when the models are attacked by simplification that causes relatively large axis error. The effect of axis error also depends on the shape of the model. If a 3D model has the spherical shape such as Head (HE) and Venus (VE), its principle components are ambiguous and the eigenvector is more sensitive to the errors of vertices. Hence, blind detection of such models yield less accurate results.

\subsection{Processing Time}

Table 6 summarizes the processing time. The resampling is used in the detection process of Praun's algorithm when the mesh is simplified and it increases the detection time only in that case. Other algorithms do not need the resampling. Registration is applied to all the non-blind algorithms when the model is modified by RST transformations. The registration time in the table is measured with the original and $1 / 2$ simplified mesh. The results show that our algorithms are much faster than Praun's and Benedens' for both embedding and detection.

\section{Conclusions}

A 3D watermarking algorithm that is robust and that supports both blind and non-blind detection is proposed in this paper. The range image is proposed to be used as the robust embedding domain. It has been shown that the range image is robust against the attacks like mesh simplification 
which changes both vertices and connectivity. Furthermore, since the embedding domain is a $2 \mathrm{D}$ image rather than a $3 \mathrm{D}$ geometry, many existing 2D image watermarking algorithms can be used for various purposes. The watermarked model can be obtained by using the explicit algorithm of vertex perturbation, which does not need any iterative optimization procedure. Thus, the proposed method requires much less computations compared to the conventional methods. The experimental results show that the robustness of the proposed algorithm is comparable to the existing methods, while its computational complexity is much lower than those of the other methods.

\section{References}

[1] M.M. Yeung, B.L. Yeo, and M. Holliman, "Digital watermarks: Shedding light on the invisible," IEEE Micro, vol.18, no.6, pp.3241, Nov. 1998.

[2] C.I. Podilchuk and E.J. Delp, "Digital watermarking: Algorithms and applications," IEEE Signal Process. Mag., vol.18, no.4, pp.3346, July 2001.

[3] H. Berghel and L. O'Gorman, "Protecting ownership rights through digital watermarking," Computer, vol.29, no.7, pp.101-103, July 1996.

[4] F. Mintzer, G.W. Braudaway, and A.E. Bell, "Opportunities for watermarking standards," Commun. ACM, vol.41, no.7, pp.57-64, July 1998.

[5] J.K. Su, F. Hartung, and B. Girod, "Digital wátermarking of text, image, and video documents," Comput. Graph., vol.22, no.6, pp.687695, Dec. 1998.

[6] R. Ohbuchi, H. Masuda, and M. Aono, "Watermarking threedimensional polygonal models through geometric and topological modifications," IEEE J. Sel. Areas Commun., vol.16, no.4, pp.551560, May 1998.

[7] R. Ohbuchi, S. Takahashi, T. Miyazawa, and A. Mukaiyama, "Watermarking 3D polygonal meshes in the mesh spectral domain," Proc. Graphics Interface 2001, 2001.

[8] R. Ohbuchi, A. Mukaiyama, and S. Takahashi, "A frequency-domain approach to watermarking 3D shapes," Computer Graphics Forum, vol.21, no.3, pp.373-382, Sept. 2002.

[9] O. Benedens, "Geometry-based watermarking of 3D models," IEEE Comput. Graph. Appl., vol.19, no.1, pp.46-55, 1999.

[10] O. Benedens, "Two high capacity methods for embedding public watermarks into 3D polygonal models," Proc. Multimedia and Security-Workshop at ACM Multimedia '99, 1999.

[11] E. Praun, H. Hoppe, and A. Finkelstein, "Robust mesh watermarking," Proc. SIGGRAPH '99, 1999.

[12] S. Kanai, H. Date, and T. Kishinami, "Digital watermarking for 3D polygons using multiresolution wavelet decomposition," Proc. Sixth IFIP WG 5.2 International Workshop on Geometric Modeling: Fundamentals and Applicattion (GEO-6) Japan 1998, 1998.

[13] B.L. Yeo and M.M. Yeung, "Watermarking 3D objects for verification,” IEEE Comput. Graph. Appl., vol.19, no.1, pp.36-45, 1999.

[14] K. Yin, Z. Pan, J. Shi, and D. Zhang, "Robust mesh watermarking based on multiresolution processing," Comput. Graph., vol.25, no.3, pp.409-420, 2001.

[15] L. Li, D. Zhang, Z. Pan, J. Shi, K. Zhou, and K. Ye, "Watermarking 3D mesh by spherical parameterization," Comput. Graph., vol.28, no.6, pp.981-989, 2004.

[16] J. Orourke, Computational geometry in C, 2nd ed., Cambridge University Press, Cambridge, UK, New York, NY, USA, 1997.

[17] R.J. Segura and F.R. Feito, "An algorithm for determining intersection segment-polygon in 3D," Comput. Graph., vol.22, no.5, pp.587-592, Oct. 1998 .

[18] A. Fujimoto and K. Iwata, "Accelerated ray tracing," Proc. Com- puter Graphics Tokyo 1985, pp.41-65, 1985.

[19] A. Fujimoto, T. Tanaka, and K. Iwata, "Arts: Accelerated ray-tracing system," IEEE Comput: Graph. Appl., vol.6, no.4, pp.16-26, April 1986.

[20] J.R. Hernandez and F. Perez-Gonzalez, "Statistical analysis of watermarking schemes for copyright protection of images," Proc. IEEE, vol.87, no.7, pp.1142-1166, July 1999.

[21] Y. Chen and G. Medioni, "Object modeling by registration of multiple range images," Image Vis. Comput., vol.10, no.3, pp.145-155, April 1992.

[22] http://graphics.stanford.edu/data/3dscanrep/

[23] http://www.cc.gatech.edu/projects/large_models/

[24] http://graphics.stanford.edu/projects/mich/

[25] P. Cignoni, C. Rocchini, and B. Scopigno, "Metro: Measuring error on simplified surfaces," Computer Graphics Forum, vol.17, no.2, pp.167-174, June 1998.

[26] G. Taubin, "A signal processing approach to fair surface design," Proc. SIGGRAPH '95, 1995.

[27] E. Paquet and M. Rioux, "Nefertiti: A query by content software for three-dimensional models databases management," Proc. International Conference on Recent Advances in 3D Digital Imaging \& Modeling 1997, 1997.

\section{Appendix A: Parameters of Bounding Cylinder}

The parameters of bounding cylinder are described here in detail. The covariance matrix of vertices can be written as

$$
\mathbf{C}=\frac{1}{n} \sum_{i=1}^{n}\left\{\left(\mathbf{v}_{i}-\mathbf{m}_{\mathbf{v}}\right)\left(\mathbf{v}_{i}-\mathbf{m}_{\mathbf{v}}\right)^{\mathbf{T}}\right\}
$$

where $\mathbf{v}_{i}$ is the three dimensional column vector of the $i$-th vertex coordinate and $n$ is the number of vertices. Three eigenvectors of $\mathbf{C}$ are used as the local axes. $\mathbf{s}_{\mathbf{1}}$ is the eigenvector corresponding to the largest eigenvalue, and $\mathbf{s}_{\mathbf{2}}$ and $\mathbf{s}_{\mathbf{3}}$ correspond to the second and third respectively. The radius $r$ and height $h$ are set to be tightly fit to the 3D model, i.e.,

$$
\begin{aligned}
& r=\max _{\mathbf{v} \in \mathcal{V}} \sqrt{\left\{\mathbf{s}_{\mathbf{2}} \mathbf{T}\left(\mathbf{v}-\mathbf{m}_{\mathbf{v}}\right)\right\}^{2}+\left\{\mathbf{s}_{\mathbf{3}} \mathbf{T}\left(\mathbf{v}-\mathbf{m}_{\mathbf{v}}\right)\right\}^{2}} \\
& h=h_{\text {max }}-h_{\text {min }}
\end{aligned}
$$

where $h_{\max }=\max _{\mathbf{v} \in \mathcal{Y}} \quad \mathbf{s}_{\mathbf{1}}^{\mathbf{T}}\left(\mathbf{v}-\mathbf{m}_{\mathbf{v}}\right)$ and $h_{\text {min }}=$ $\min _{\mathbf{v} \in \mathcal{Y}} \quad \mathbf{s}_{\mathbf{1}} \mathbf{T}\left(\mathbf{v}-\mathbf{m}_{\mathbf{v}}\right)$. Note that $h_{\max }$ is positive and $h_{\min }$ is negative.

The signs of eigenvectors can be different depending on the eigenvalue decomposition methods, even though the given data are the same. In order to have consistent directions, we exploit the distribution of vertices described in [27],

$$
D_{\mathcal{X}}=\sqrt{\frac{1}{n} \sum_{\mathbf{x} \in \mathcal{X}}\left\|\mathbf{x}-\mathbf{m}_{\mathcal{X}}\right\|^{2}}
$$

where $\mathbf{m}_{X}$ is the average of all the vectors in a set $\mathcal{X}$. To make $\mathbf{s}_{1}$ consistent, the set of vertices is divided into two sets $\mathcal{A}$ and $\mathcal{B}$ such that

$$
\begin{aligned}
& \mathcal{A}=\left\{\mathbf{v}: \mathbf{v} \in \mathcal{V} \text { and } \mathbf{s}_{\mathbf{1}} \mathbf{T}\left(\mathbf{v}-\mathbf{m}_{\mathbf{v}}\right)>0\right\} \\
& \mathcal{B}=\left\{\mathbf{v}: \mathbf{v} \in \mathcal{V} \text { and } \mathbf{s}_{\mathbf{1}} \mathbf{T}\left(\mathbf{v}-\mathbf{m}_{\mathbf{v}}\right)<0\right\}
\end{aligned}
$$


where $\mathcal{V}$ is the set of all the vertices. If $D_{\mathcal{A}}<D_{\mathcal{B}}$, the direction of $\mathbf{s}_{\mathbf{1}}$ is reversed. If not, the direction remains the same. For the vector $\mathbf{s}_{\mathbf{2}}$, the same procedure is performed for its consistency. $\mathbf{s}_{\mathbf{3}}$ is just defined as $\mathbf{s}_{\mathbf{3}}=\mathbf{s}_{\mathbf{2}} \times \mathbf{s}_{\mathbf{1}}$. Then, $\mathbf{s}_{\mathbf{1}}, \mathbf{s}_{\mathbf{2}}$ and $\mathbf{s}_{\mathbf{3}}$ are normalized to be unit vectors.

\section{Appendix B: Decision of $N_{u}$ and $N_{v}$}

The method to decide the size of the range image $\left(N_{v} \times N_{u}\right)$ is described here. If $N_{u}$ and $N_{v}$ are too large, the $u-v$ grid becomes too dense and some of $\mathcal{B}\left(u_{r}, v_{r}\right)$ in Eq. (9) may have no vertex in it, which means that the watermark cannot be embedded in this region. Hence, it should be guaranteed that $\mathcal{B}\left(u_{r}, v_{r}\right)$ contains a certain amount of vertices. For this, we need to estimate how many vertices will be included in each set. However, since it is difficult to figure out the density of vertices in general cases, we first consider the ideal case of an infinite plane of equilateral triangular mesh. In this mesh, each vertex is the center of equilateral hexagon. The smallest hexagon is denoted as 1-ring of a vertex. The number of triangles in the 1-ring is six and the number of the vertices inside it is 1 . Let the next bigger equilateral hexagon centered at the same vertex be the 2-ring and so on. Let the number of vertices and triangles in the $n$-ring be $V_{n}$ and $T_{n}$ respectively. Then, they can be expressed as

$$
\begin{aligned}
& V_{n}=1+6 \sum_{i=1}^{n}(i-1) \\
& T_{n}=6 \sum_{i=1}^{n}(2 i-1) .
\end{aligned}
$$

It can also be easily shown that the area of the $n$-ring is

$$
S_{n}=\frac{\sqrt{3} a^{2}}{4} T_{n}
$$

where $a$ is the edge length of the equilateral triangle. Then, the asymptotic density of the vertices, i.e., the number of vertices per unit area can be defined as

$$
\lim _{n \rightarrow \infty} \frac{V_{n}}{S_{n}}=\frac{2}{\sqrt{3} a^{2}} .
$$

This result cannot be applied to our problem directly, because a $3 \mathrm{D}$ model is not an infinite plane and the triangles are not equilateral. But when the mesh density is very high, the above results can be applied by using the average edge length instead of $a$ in Eq. (A.7). That is,

$$
a=\bar{E}+d \sigma_{E}
$$

where $\bar{E}$ is the mean length of the edges, $\sigma_{E}$ is the standard deviation of the length and $d$ is a constant that gives more margin. Then, the number of vertices included in a $\Delta u \times \Delta v$ rectangular region is

$$
\frac{2}{\sqrt{3}\left(\bar{E}+d \sigma_{E}\right)^{2}} \Delta u \wedge v,
$$

where $\Delta u$ and $\Delta v$ are the height and width of the rectangular region respectively. Now, let $\Delta u$ and $\Delta v$ be the height and width of the region for $\mathcal{B}\left(u_{r}, v_{r}\right)$. Then these are related to the $N_{u}$ and $N_{v}$ as

$$
\begin{aligned}
\Delta u & =\frac{h}{N_{u}} \\
\Delta v & =\frac{2 \pi r_{\text {avg }}}{N_{v}},
\end{aligned}
$$

where $r_{\text {avg }}$ is the mean radius of vertices. In this paper, we set $\Delta u$ and $\Delta v$ as the multiples of average edge length, that is,

$$
\Delta u=\Delta v=c\left(\bar{E}+d \sigma_{E}\right)
$$

Then, the number of vertices within this $\Delta u \times \Delta v$ rectangular region can be calculated by Eqs. (A.9) and (A. 12) as

$$
\frac{2 c^{2}}{\sqrt{3}}=1.15 c^{2} \text {. }
$$

This means that the set $\mathcal{B}\left(u_{r}, v_{r}\right)$ contains approximately $1.15 c^{2}$ vertices. Actually, $\mathcal{B}\left(u_{r}, v_{r}\right)$ contains more than $1.15 c^{2}$ vertices because the constant $d$ gives more margin. Finally, the $N_{u}$ and $N_{v}$ can be decided by using Eqs. (A. 10), (A. 11) and (A. 12) as

$$
\begin{aligned}
& N_{u}=\frac{h}{c\left(\bar{E}+d \sigma_{E}\right)} \\
& N_{v}=\frac{2 \pi r_{a v g}}{c\left(\bar{E}+d \sigma_{E}\right)} .
\end{aligned}
$$

These are not the exact solutions due to the assumptions that we have made. However, they can be used to set some initial values for $N_{u}$ and $N_{v}$, and the approximate upper bound can be estimated by setting $c=1$ and $d=0$. In our experiments, $N_{u}$ and $N_{v}$ are decided by using Eqs. (A. 14) and (A. 15) with $c=2$ and $d=2$.

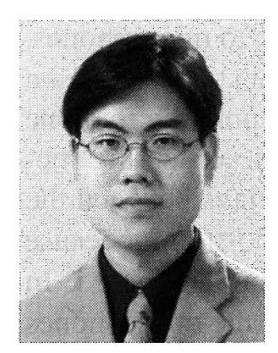

Han Sae Song received B.S. and M.S. degrees in electrical engineering from Seoul National University, Seoul, Korea, in 2001 and 2003 , respectively. He is currently working toward the $\mathrm{Ph} . \mathrm{D}$. degree in electrical engineering at Seoul National University. His research interests include image, video signal processing and information security.

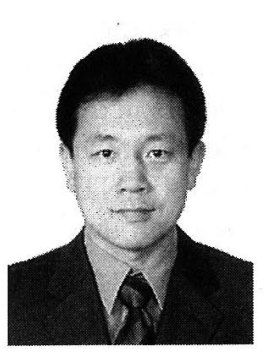

Nam Ik Cho received the B.S., M.S., and $\mathrm{Ph} . \mathrm{D}$. degrees in control and instrumentation engineering from Seoul National University, Seoul, Korea, in 1986, 1988, and 1992, respectively. From 1991 to 1993, he was a Research Associate of the Engineering Research Center for Advanced Control and Instrumentation, Seoul National University. From 1994 to 1998 , he was with the University of Seoul, Seoul, Korea, as an Assistant Professor of Electrical Engineering. He joined the School of Electrical Fngineering, Seoul National University, in 1999, where he is currently a Professor. His research interests include speech, image, video signal processing, and adaptive filtering. 